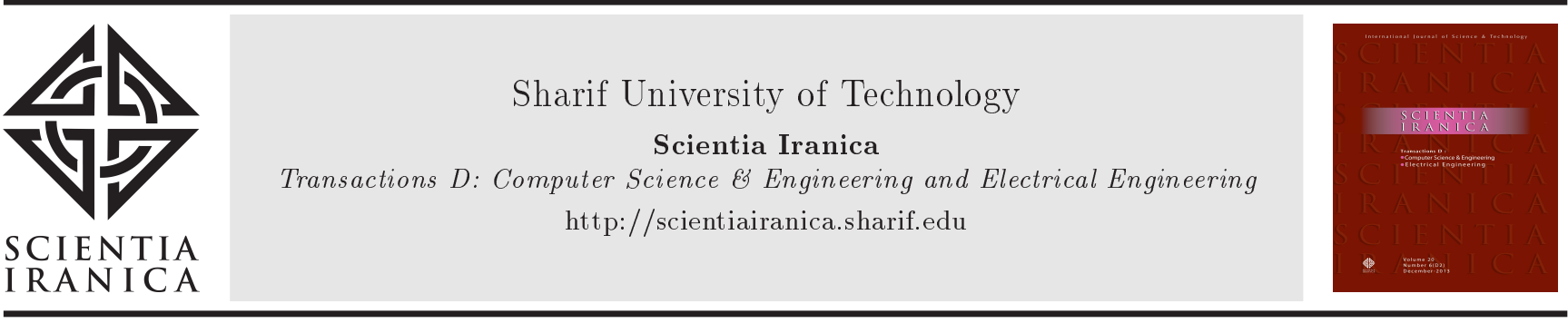

\title{
A DC power system stabilizer based on passivity- oriented DC bus impedance shaping
}

\author{
A. Asbafkan* and H. Mokhtari \\ Department of Electrical Engineering, Sharif University of Technology, Tehran, Iran.
}

Received 3 June 2020; received in revised form 24 July 2020; accepted 16 November 2020

\section{KEYWORDS}

Dynamic interaction;

Passivity;

Power system

stabilizer;

System identification.

\begin{abstract}
High penetration of Power Electronic (PE) converters in DC power grids causes new stability challenges due to dynamic interactions among the subsystems of a network. Such dynamic interactions can be avoided by the impedance coordination among the subsystems through the modification of control loops or passive elements inside a grid. Impedance coordination is a very complex and time-consuming task with no adaptations to dynamic changes in a power grid. The current study delved into the concepts of dynamic interaction and passivity and they were combined to provide an online stability measure concerning the DC bus impedance characteristics. In this regard, a novel DC Power System Stabilizer (PSS) was proposed, which was connected to a DC bus as a separate module passivizing the bus impedance at non-passive interaction frequencies. The interaction frequencies were detected through a broadband online identification process. The PSS working principle, topology, modeling, and control designs were also discussed in detail. Finally, the functionality and performance of the proposed stabilizer were validated by the simulation results.

(C) 2022 Sharif University of Technology. All rights reserved.
\end{abstract}

\section{Introduction}

Medium Voltage DC (MVDC) grid technology has received a great deal of attention over the past decade when applied to electric power distribution systems and micro grids. DC grids are beneficial due to their high power-density, efficiency, less harmonic pollution, fast dynamic responses, and better integration into renewable energy sources and energy storage systems. DC technology has a wide range of applications in railway [1], aerospace [2], marine, and offshore industries [3]. In addition, it is still developed for the expansion of the grid infrastructure at transmission and distribution levels [4].

DC power distribution systems are highly pen-

*. Corresponding author. Tel.: +982166165962 E-mail addresses: a.turbodesign@gmail.com (A. Asbafkan); mokhtari@sharif.edu(H. Mokhtari)

doi: $10.24200 /$ sci. 2020.56115 .4556 etrated by Power Electronic (PE) converters that are characterized by non-linear dynamics. In practice, these converters are designed to ensure high dynamic performance with optimized stability margins [5]. However, when they are connected to each other in a multi-converter system, new stability challenges are likely to face. Due to the tight regulation of the control loops, a non-minimum phase dynamic is introduced at the input terminal of the PE loads which can cause instability [6]. This phenomenon is regarded as Constant Power Load (CPL) destabilizing effect. However, the CPL behavior is not the worst-case scenario, and some incidents of instability under light load conditions have been also reported in industries mainly due to the interactions among the control loops of the interconnected converters [7]. In this case, the instability arises from a totally different phenomenon other than the CPL effect; yet, both result in negative impedance at the input terminal of the PE loads [8].

The history of the stability analysis for multiconverter systems dates back to the Middlebrook's 
work on filter design [9] and its Nyquist derived extensions to the Gain and Phase Margin Criterion, Opposing Argument Criterion, and Energy Source Analysis Consortium (ESAC) Criterion, all reviewed and compared in [10]. These criteria have long been used for the impedance coordination among PE converters in aerospace [2] and marine industries [11]. These criteria specify different forbidden regions in the polar plot of the so-called Minor Loop Gain (MLG), which is the impedance ratio of the source subsystem to that of the load subsystem. Impedance coordination is a very complex and time-consuming task functioning based on the frequency response measurement of each subsystem at different operating points $[2,12]$. Unfortunately, these criteria that result in a conservative design are sensitive to components grouping, system uncertainties, load conditions, and power flow direction in a network [10].

Recently, a novel Passivity-Based Stability Criterion (PBSC) has been proposed based on the passivity of the overall DC bus impedance from any arbitrary point [13]. In addition, there are some earlier research works on the passivity of the overall DC bus admittance developed for railway industries that led to Input Admittance Criterion (IAC) [14] and EN50388 standard [15]. Of note, the observability of an unstable mode from any arbitrary point in a power network is still an open controversial problem; however, initial investigations into the practical power systems indicate that any unstable mode is propagated throughout the grid without any zero/pole cancellation [14].

A number of stabilization techniques have been proposed in the literature among which active damping methods are more preferred to others due to their higher efficiency and lower costs. General approaches of active damping methods are to modify the impedance characteristics of the PE converters on either the load or source sides to fulfil the stability criteria. Virtual impedance techniques [16,17], passivity-oriented admittance shaping [18], and DC bus voltage feedforwarding [19] are some general methods of active damping applied to the load-side converters. Other solutions such as feedback linearization [20], backstepping control [21], synergetic control [22], and multi-agent stabilizing control [23] are the nonlinear stabilizing techniques that can provide large signal stability. It should be noted that nonlinear solutions are complex and highly dependent on the system model and parameters. A passivity-based stabilizing control was proposed in [24] which added a supplementary positive feed-forward loop to the load-side converter. However, the control design is highly computational and dependent on the converter unterminated model and transfer functions. In [25], a passivity-based stabilizing control was implemented on the sourceside converter which was very simple in design and calculation. In this method, a notch filter was added to the voltage control loop to enhance the DC bus stiffness at the interaction frequency. However, the bandwidth of the voltage loop was limited, thus making the implementation of the notch filter almost impossible in a wide frequency range.

All the aforementioned stabilizing methods, whether they were applied to the load-side or the source-side, impose oscillatory damping power disturbances on the network subsystems, leading to their performance degradation or cyclic stress increment at their mechanical drive trains. Of note, such control modifications cannot be applied to commercial PE converters with a limited access to their firmware. These shortcomings call for a centralized stabilizer in a DC grid.

The idea of centralized stabilizer was proposed in [26] for modification of the source-side impedance. However, in practice, the source-side converter is not decoupled from the rest of the network, and the explicit form of its output impedance is not known for the stabilizer. Another centralized stabilizer was proposed in [27] which employed Second-Order-GeneralizedIntegrator (SOGI) to shape its output impedance around the specific resonance frequency. However, due to the lagging function of the SOGI filters at higher frequencies than notch frequencies, an inductor-like impedance was introduced to the grid, which would result in new unexpected LC resonance modes.

The current study introduced a DC Power System Stabilizer (PSS) connected to a DC bus as a separate module. The PSS operates on the principle of shaping the DC bus impedance with the enforcement of the passivity condition at non-passive interaction frequencies. The interaction frequencies were detected through a broadband impedance identification process done by local measurements. The proposed approach enjoys several advantages namely the centralized implementation with no modifications to the grid structure in terms of software, hardware or communication related aspects, well-structured design and simple calculations, and adaptation to the network dynamic changes with a fixed control structure, to name a few.

This paper is organized as follows: Section 2 introduces the concept of dynamic interaction among $\mathrm{PE}$ converters and combines them with the PBSC to provide guidelines for the stability assessment of DC grids in terms of equivalent bus impedance. Section 3 discusses the PSS working principle, functionality, and sequences. Section 4 models the PSS and designs its control loops. Finally, Section 5 evaluates the PSS performance using simulation results.

\section{Dynamic interaction and stability analysis}

Power systems with high penetration of $\mathrm{PE}$ converters are prone to voltage oscillations or instabilities. $\mathrm{PE}$ 
converters are controlled-devices with nonlinear dynamics which, in a small signal sense, are modeled by complex transfer functions in the canonical form [28]. The terminal characteristics of the PE converters are attributed to their control parameters, operating point, and filter components. A DC power distribution system can be considered as an interconnection between an upstream source and a downstream load subsystem. As depicted in Figure 1, the source and load subsystem impedances are denoted by $Z_{S}$ and $Z_{L}$, respectively. The equivalent DC bus impedance is:

$$
Z_{\text {bus }}=\frac{Z_{S} \cdot Z_{L}}{Z_{S}+Z_{L}}=Z_{S}\left[\frac{1}{1+Z_{S} / Z_{L}}\right] .
$$

According to Eq. (1), the bus impedance is represented by $Z_{S}$ multiplied by a correction factor which is a function of $Z_{S} / Z_{L}$ (MLG). The network is considered stable if $Z_{S}$ and $Z_{L}$ are individually stable, and the Nyquist contour of the correction factor is not encircled $(-1,0)$. In practice, $Z_{S}$ dominates the bus impedance in low frequency ranges where the bandwidth of the source subsystem is wide enough to support the load dynamics. On the contrary, in high frequency ranges, the bus impedance is dominated by the source-side capacitor banks. In both low and high frequency ranges, the MLG magnitude is very small, hence $Z_{\text {bus }} \approx Z_{S}$.

Dynamic interaction may occur in a midfrequency range where the bandwidth of the sourceside is limited, hence $Z_{S}$ and $Z_{L}$ are comparable in magnitude but with an obtuse angle. The $Z_{b u s}$ peaking phenomenon occurs when the phase difference of $Z_{S}$ and $Z_{L}$ exceeds $120^{\circ}$. For the phase differences of more than $150^{\circ}$, the amount of resonance peak exceeds $6 \mathrm{~dB}$, and the associated bus ringing and performance degradation occur due to the amplification effect of the correction factor [2]. As compared in Figure 2, this condition is similar to the resonance phenomenon with a simple difference that the feedback control loops interact with each other, and either $Z_{S}$ or $Z_{L}$ exhibits a negative real-part which reduces network damping.

Any resonance peak in $Z_{b u s}$, whether it was originated from the interaction of the physical LC components in the grid or from the PE converters control loops, is the representative of the network instability. The system stability at a resonance peak depends on the net damping of a power grid. Depending on the passivity condition of $Z_{b u s}$, the sign of the net damping

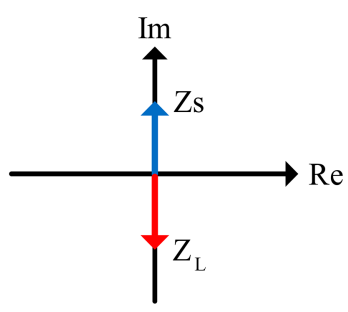

Resonance

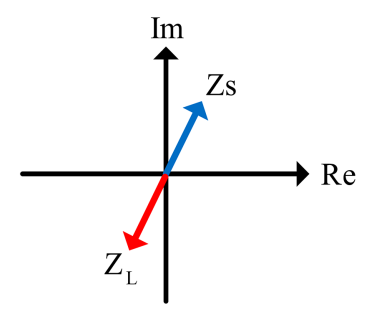

Dynamic Interaction
Figure 2. Vector plots of $Z_{S}$ and $Z_{L}$ in case of resonance and dynamic interaction.

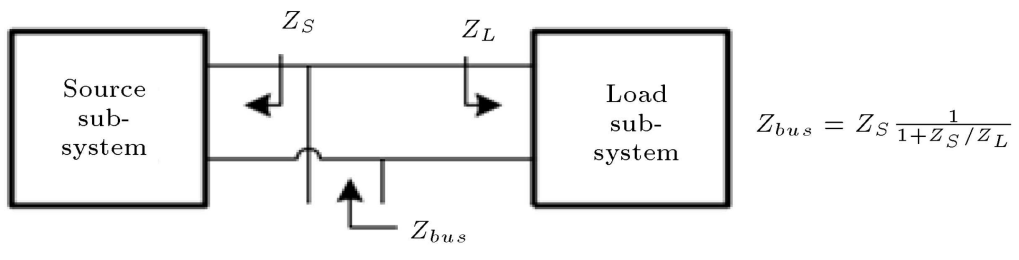

(a)
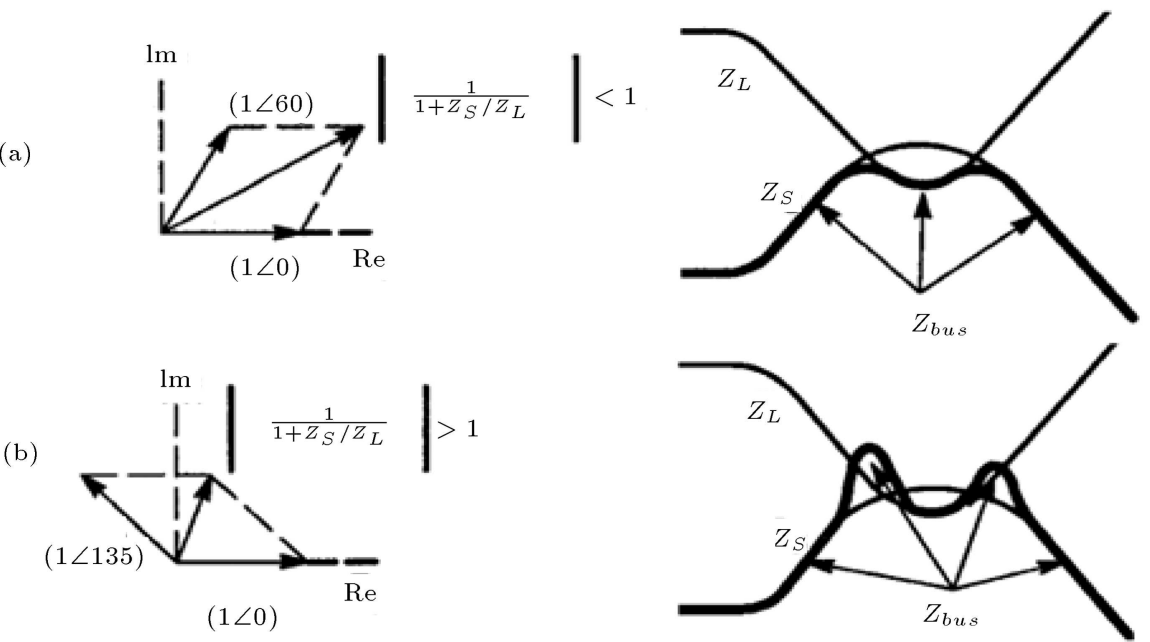

Figure 1. $Z_{\text {bus }}$ peaking phenomenon at an overlap frequency caused by the amplification effect of the correction factor $\left(1 / 1+Z_{S} / Z_{L}\right)$ : (a) Angle of $Z_{S} / Z_{L}$ lower than $120^{\circ}$ and (b) angle of $Z_{S} / Z_{L}$ exceeding $120^{\circ}$ [2]. 
can be determined. Network instability is identified when the $Z_{\text {bus }}$ phase angle varies around $180^{\circ}$ at a resonant peak. Preventive actions can be taken prior to the beginning of the oscillations when the phase angle is still less than $180^{\circ}$.

In a small signal sense, a DC bus is passive if $Z_{\text {bus }}$ is a positive real transfer function [29]; in other words:

$$
\operatorname{Re}\left[Z_{b u s}(\sigma+j \omega)\right] \geq 0, \quad \forall \sigma>0, \forall \omega .
$$

Evaluation of the positive real property in the Right-Half-Plane (RHP) is a very complex and formidable task. According to the real part corollary of the maximum modulus theorem [30], the minimum value in Eq. (2) can be obtained on the boundary of the RHP, i.e., the imaginary axis, provided that there is no singularity inside the RHP region. Therefore, the bus impedance is passive if and only if:

- $Z_{\text {bus }}$ (s) has no RHP poles;

- and the Nyquist contour of $Z_{b u s}(j \omega)$ completely lies in the RHP.

The first condition stands for the system stability; however, the degree of passivity is higher than that of stability. Passivity can bring about robust stability which is important in practical systems with delays and uncertainties [13]. The RHP Nyquist contour can be evaluated by the following phase constraint:

$$
-\frac{\pi}{2} \leq \arg \left(Z_{\text {bus }}(j \omega)\right) \leq \frac{\pi}{2}, \quad \forall \omega .
$$

Passivity is a sufficient condition for the network stability, indicating that a system is considered stable even if there are some non-passive frequency ranges in the $Z_{\text {bus }}$ spectrum. Therefore, it is not required to check the passivity condition in the whole frequency ranges.

\section{Proposed stabilizing method}

The main idea behind the proposed stabilizer is to enforce the passivity condition to $Z_{\text {bus }}$ at non-passive resonance frequencies. The task can be actively accomplished by modifying the bus impedance around the resonance frequency in accordance with the satisfaction of the passivity phase constraint in Eq. (3). As shown in Figure 3, the DC PSS injects a damping current ( $\left.I_{\text {damp }}\right)$ to the DC bus which emulates virtual damping impedance $\left(Z_{\text {damp }}\right)$ that is parallel to $Z_{\text {bus }}$. The damping impedance is designed to be passive and dominant with respect to the DC bus impedance $\left(Z_{\text {damp }}<<\right.$ $\left.Z_{\text {bus }}\right)$ at the resonance frequency. Therefore, the total bus impedance $\left(Z_{\text {bus_new }}\right)$ bears some similarity with $Z_{\text {damp }}$ characteristics, being passive and thus stable. The amplitude of the damping current is proportional to the DC bus voltage oscillations and decreases by zero as the grid voltage oscillations damp.

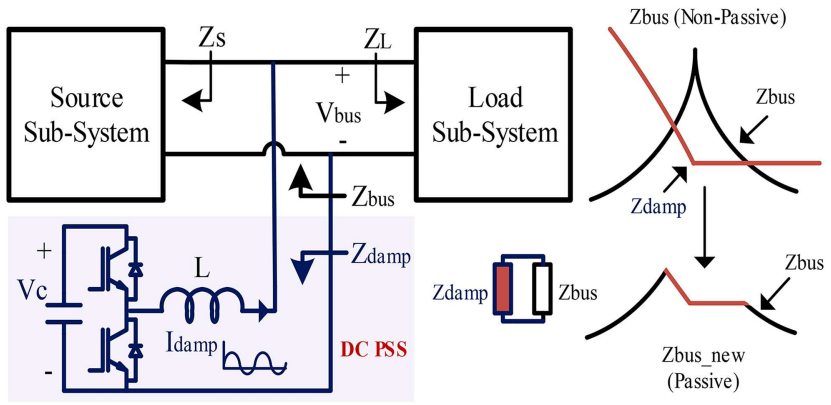

Figure 3. DC bus impedance shaping at an interaction frequency based on the proposed DC PSS. The DC bus impedance is modified to $Z_{\text {bus-new }}$ by adding virtual damping impedance $\left(Z_{\text {damp }}\right)$ in parallel to the grid impedance $\left(Z_{\text {bus }}\right)$.

The proposed stabilization method does not depend on the explicit transfer function, $Z_{\text {bus }}$. Instead, it is directly dependent on the $Z_{b u s}$ frequency spectrum achieved from the non-parametric online identification. Wideband identification of $Z_{b u s}$ is done by perturbing the grid voltage with a Pseudo-RandomBinary-Sequence (PRBS) signal. The PRBS signal is a digital approximation of the white noise that excites all frequencies of interest over a fraction of time [31]. The DC bus impedance frequency spectrum is obtained, followed by applying the Discrete-Fourier-Transform (DFT) to the perturbed signals [32]. Based on the identified $Z_{b u s}$, the non-passive resonance peak (if any) is detected and the required $Z_{\text {damp }}$ is calculated. Of note, the identification process can be conditionally activated as the DC bus voltage deviates from a predefined threshold. However, as far as the online stability monitoring and preventive actions are concerned, the $\mathrm{DC}$ bus can be identified all the time. The flowchart of the stabilizing process is shown in Figure 4.

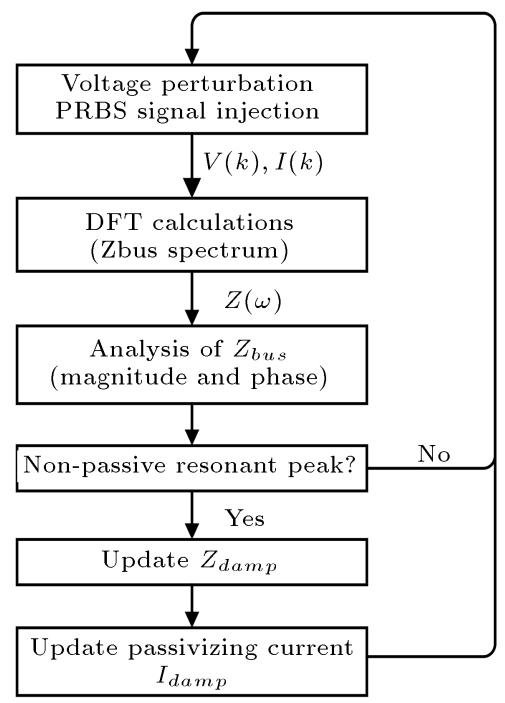

Figure 4. Flowchart of the stabilizing process implemented by the DC PSS. 


\section{DC PSS structure and control design}

As shown in Figure 5, the PSS involves two power circuits namely the stabilizing and perturbing circuits. The stabilizing circuit injects the damping current to the grid while the perturbing circuit is responsible for exciting a network with the PRBS signal. Both circuits are implemented in a half-bridge topology. The DC PSS capacitor voltage is regulated by the stabilizer at a higher level than that of the grid voltage $\left(V_{C}>V_{\text {bus }}\right)$. The higher voltage is required for the bidirectional current control capability and provision of a duty cycle margin to avoid the modulator saturation in the perturbing circuit.

The control structure of the stabilizing circuit comprises the cascaded voltage and current control loops. The stabilizer reference current is tracked by an internal control loop and the PSS capacitor voltage (VC) is regulated by an external control loop.

The control loop of the perturbing circuit regulates the current at nil in order to block the circulating current between the two half-bridge converters. The PRBS signal is added to the modulator of the perturbing circuit and feed-forwarded to the reference signal to ensure that it is not rejected by the control action in low frequency ranges. The stabilizer reference current calculation, modeling, and control design are explained in the following sections:

\subsection{Calculation of the passivizing reference current}

The damping impedance $\left(Z_{\text {damp }}\right)$ should:

- Exhibit high impedance in low frequency ranges;

- Exhibit a dissipative (passive) behavior in the resonance frequency range;

- Not exhibit an inductive behavior at higher frequencies than the interaction frequency in order to avoid unexpended LC resonance between the stabilizer and grid.

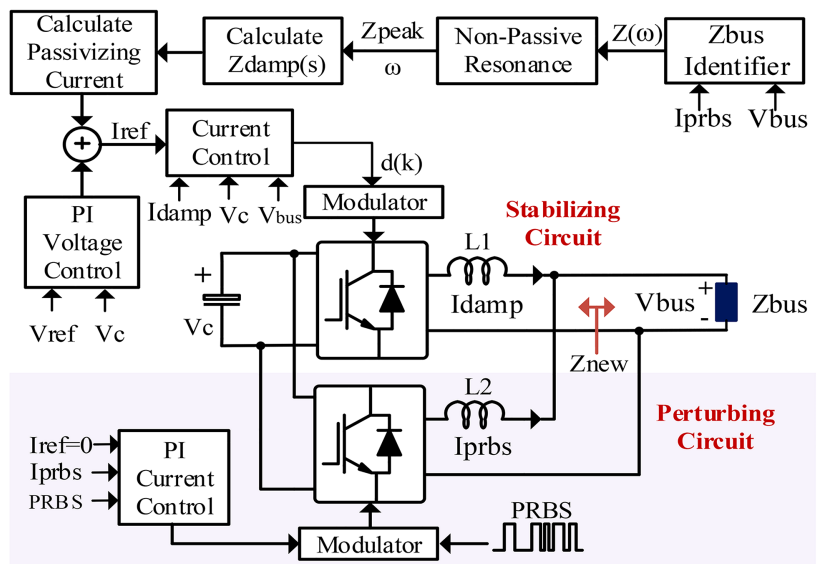

Figure 5. DC PSS power circuit and control block diagrams.
$Z_{\text {damp }}$ is designed to be a series RC-branch with parameters tuned based on the identified $Z_{b u s}$ at the resonance frequency. The $\mathrm{RC}$-branch corner frequency is designed to be one decade below the resonance frequency (fres) with the impedance value to be one order of magnitude smaller than the grid impedance peak value at the resonance frequency $\left(Z_{\text {peak }}\right)$. Therefore, the parameters of the $\mathrm{RC}$-branch are:

$$
R=\frac{Z_{\text {peak }}}{10}, \quad C=\frac{10}{2 \pi R_{\text {fres }}} .
$$

The stabilizer reference current is calculated by Eq. (5):

$$
I_{\text {damp }}(s)=\frac{V_{\text {bus }}(s)}{Z_{\text {damp }}(s)}=\frac{V_{b u s}(s)}{\left(R+\frac{1}{C S}\right)},
$$

where " $s$ " denotes the Laplace variable.

Based on the Backward Euler discretization method with a time step of $T_{S}$, the discrete form of the passivizing current can be obtained as follows:

$$
\begin{aligned}
I_{d a m p}(k)= & \left(\frac{C}{T S+R C}\right) \cdot\left[V_{b u s}(k)-V_{\text {bus }}(k-1)\right] \\
& +\left(\frac{R C}{T S+R C}\right) \cdot I_{\text {damp }}(k-1),
\end{aligned}
$$

where " $k$ " denotes the $k$ th control iteration.

\subsection{Current control loop}

A deadbeat current controller was utilized to track the calculated reference current with fast dynamic response [30]. In this control method, the actual current converges to the reference value after two sample time delays $\left(z^{-2}\right)$. The control rule was derived from [33] and represented here in terms of the stabilizer DC link voltage $\left(V_{C}\right)$, reference current $\left(I_{\text {ref }}\right)$, actual current $\left(I_{L}\right)$, grid voltage $\left(V_{\text {bus }}\right)$, and converter switching frequency $\left(f_{S W}\right)$. The switching duty-cycle $(d)$ of the stabilizer circuit modulator is:

$$
\begin{aligned}
d(k+1)= & -d(k)+\left(I_{\text {ref }}(k)-I_{L}(k)\right) \frac{L \cdot f_{S W}}{V_{C}(k)} \\
& +2 \frac{V_{\text {bus }}(k)}{V_{C}(k)} .
\end{aligned}
$$

\subsection{Voltage control loop}

The DC PSS voltage control can be obtained through the stabilizer current control. The average model of the half-bridge topology was taken into account to find out the DC PSS dynamics, as shown below:

$$
C \frac{d V_{C}}{d t}=-d . I_{d a m p}-d . I_{P R B S}
$$

where $C$ and $V_{C}$ are the stabilizer DC link capacitance 


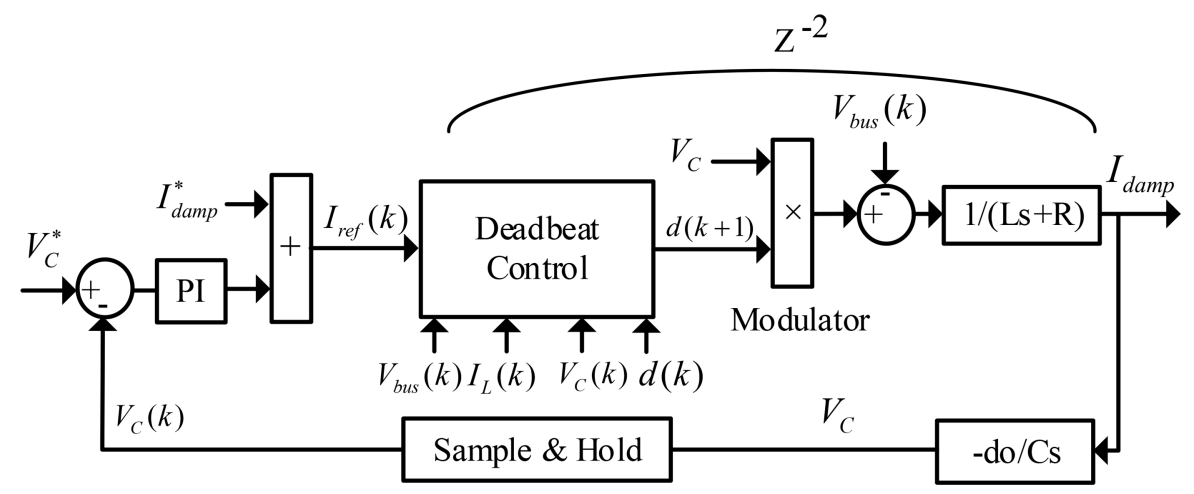

Figure 6. The stabilizer model and control hierarchy.

and voltage, respectively. The small-signal linearization of Eq. (8) is:

$$
\begin{aligned}
-C \frac{d \tilde{V}_{C}}{d t}= & d_{O} \cdot \tilde{I}_{d a m p}+\tilde{d} . I_{d a m p O}+\tilde{d} \cdot \tilde{I}_{d a m p O} \\
& + \text { dist.term }
\end{aligned}
$$

where " " and "O" denote the small signal variable and operating point, respectively. The perturbing circuit does not participate in the PSS voltage control and consequently, the $I_{P R B S}$ is modeled as a disturbance term (dist.term) in Eq. (9).

The second-order signal perturbation in Eq. (9) was neglected; consequently, the transfer function from the stabilizer output current to the DC link capacitor voltage can be written as follows:

$$
\frac{\tilde{V}_{C}(S)}{\tilde{I}_{\text {damp }}(S)}=\frac{-d_{O}}{C S} .
$$

In order to regulate $V_{C}$ at the reference value, a Proportional-Integral (PI) controller should be used:

$$
P I(S)=K_{P}\left(1+\frac{1}{T_{I} S}\right),
$$

where $K_{P}$ is the proportional gain and $T_{I}$ the integration time constant.

The $P I$ controller parameters are tuned using the Symmetrical Optimum Criterion (SOC) [5]. Figure 6 presents the stabilizer model and control hierarchy, and Figure 7 shows the root locus of the voltage loop. The PSS power circuit ratings and control parameters are listed in Table 1.

\section{Simulation results}

Figure 8 introduces a DC power distribution system of the cascaded source and load subsystems. The power system consists of an Active-Front-End (AFE) rectifier as the source subsystem and a metro traction motor drive as the load subsystem. The simulations

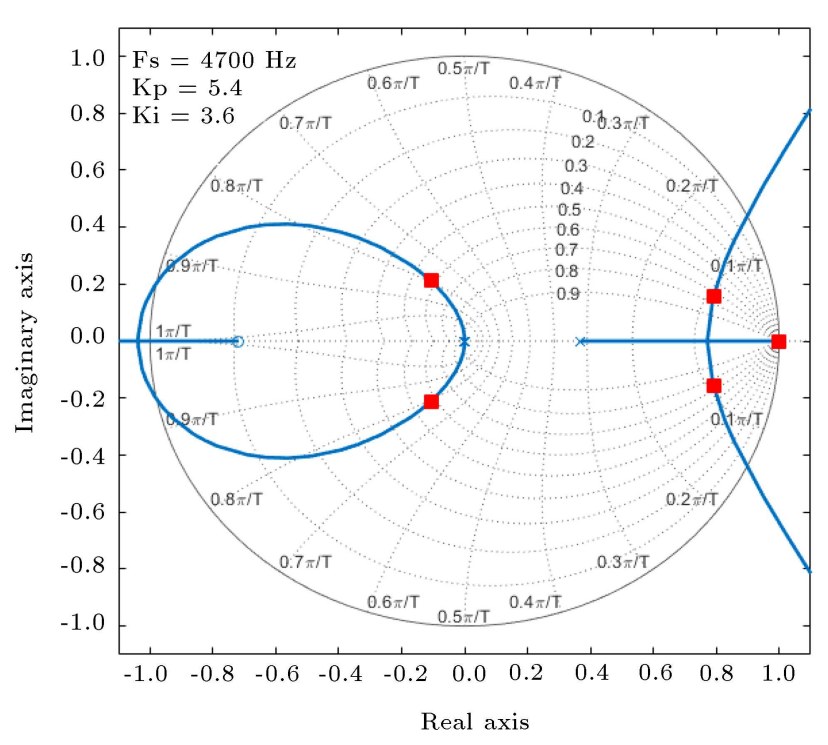

Figure 7. Root locus of the stabilizer voltage control loop.

Table 1. Control parameters and ratings for DC PSS.

\begin{tabular}{lcc}
\hline Description & Symbol & Value \\
\hline Stabilizing circuit filter inductance & $\mathrm{L} 1$ & $2 \mathrm{mH}$ \\
Perturbing circuit filter inductance & $\mathrm{L} 2$ & $1 \mathrm{mH}$ \\
DC link capacitance & $\mathrm{C} 3$ & $4 \mathrm{mF}$ \\
Voltage loop PI parameters & $K_{P}, T_{i}$ & $5.4,1.5 \mathrm{sec}$ \\
Perturbing circuit PI parameters & $K_{P}, T_{i}$ & $3,10 \mathrm{sec}$ \\
PRBS generation frequency & $F_{0}$ & $4700 \mathrm{~Hz}$ \\
PRBS bits & - & $10 \mathrm{bits}$ \\
Switching frequency & $F_{S W}$ & $4700 \mathrm{~Hz}$ \\
Control sampling frequency & $F_{S}$ & $4700 \mathrm{~Hz}$ \\
\hline
\end{tabular}

were performed in MATLAB/Simulink environment at a time step of $2 \times 10^{-6} \mathrm{~s}$.

The current control loops of the AFE rectifier and traction drive are designed based on the Optimum Modulus criteria [5]. The bandwidth of the AFE rectifier is intentionally reduced and as a result, a dynamic interaction between the two subsystems occurs within 


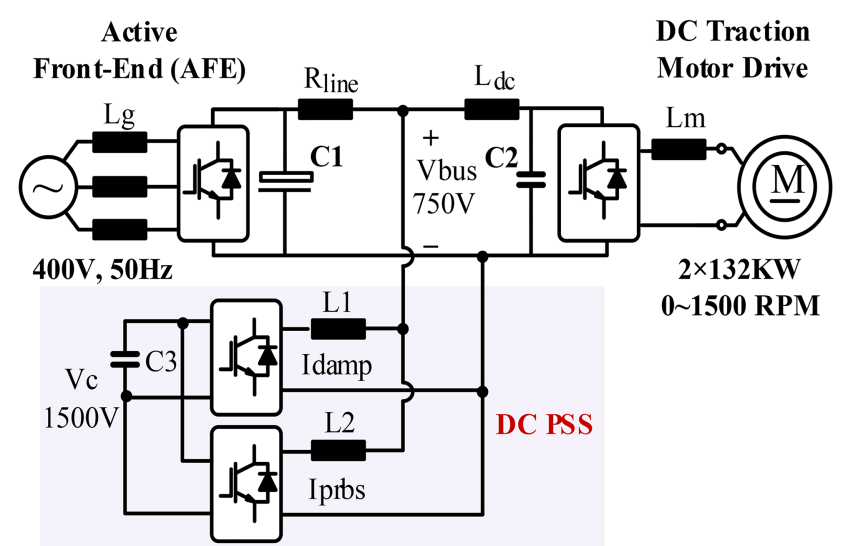

Figure 8. Power circuit of the system under study.

Table 2. Parameters of the DC power distribution system.

\begin{tabular}{lcc}
\hline Description & Symbol & Value \\
\hline AFE rectifier & & \\
Input filter & $L_{g}, R_{g}$ & $2 \mathrm{mH}, 0.1 \Omega$ \\
DC link capacitance & $\mathrm{C} 1$ & $15 \mathrm{mF}$ \\
Current loop PI parameters & $K_{P}, T_{i}$ & $2.8,0.05 \mathrm{sec}$ \\
Voltage loop PI parameters & $K_{P}, T_{i}$ & $9,0.1 \mathrm{sec}$ \\
PLL loop PI parameters & $K_{P}, T_{i}$ & $4.6,0.5 \mathrm{sec}$ \\
Switching frequency & $F_{S W}$ & $3000 \mathrm{~Hz}$ \\
Line resistance & $R_{l i n e}$ & $0.05 \Omega$ \\
\hline Motor drive & & \\
Input filter inductance & $L_{d c}$ & $1.2 \mathrm{mH}$ \\
Input filter capacitance & $\mathrm{C} 2$ & $1.5 \mathrm{mF}$ \\
Output filter inductance & $L_{m}$ & $4 \mathrm{mH}$ \\
Current loop PI parameters & $K_{P}, T_{i}$ & $9.3,0.04 \mathrm{sec}$ \\
Switching frequency & $F_{S W}$ & $3600 \mathrm{~Hz}$ \\
\hline
\end{tabular}

the nominal power range of the grid. Table 2 lists the parameters of the AFE rectifier and traction motor drive. The PRBS parameters are tuned based on the system noise level, degree of non-linearity, time constants, and desired frequency resolution [34]. The PRBS wave was generated in this study by a $4700 \mathrm{~Hz}$ 10-bit Linear-Feedback-Shift-Register (LFSR) with a resolution of $4.6 \mathrm{~Hz}$. The PRBS signal amplitude was designed large enough to excite the DC bus dynamics in the whole frequency ranges and small enough to reduce the small-signal modeling errors originated from the system non-linearity. In practice, the PRBS amplitude was designed to perturb the DC bus voltage around $3 \%$ to $5 \%$.

In the first simulation, the stability of the power grid was investigated in different load conditions. The
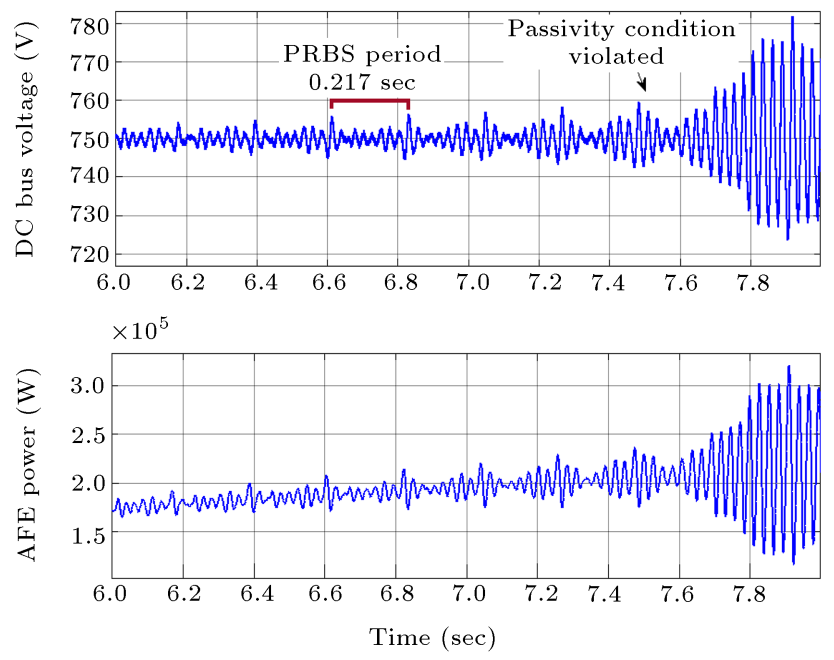

Figure 9. DC bus voltage (top) and power (bottom) at the terminal of the AFE rectifier. Oscillations start at $200 \mathrm{~kW}$ load power.

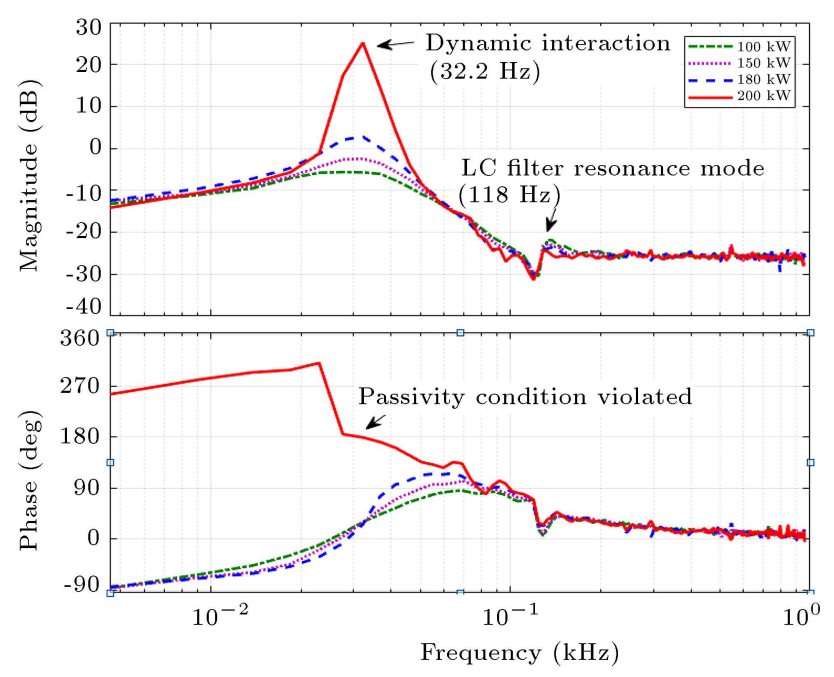

Figure 10. Bode plot of $Z_{b u s}$ at different load conditions.

DC bus voltage is regulated at $750 \mathrm{~V}$ by the AFE rectifier, and the traction motors are accelerated with a constant torque. The PSS is connected to the DC bus; however, only the perturbing circuit is operational in terms of stability monitoring. Figure 9 shows the DC bus voltage and power at the rectifier terminal. As observed in this figure, an unstable oscillation begins at a load power of $200 \mathrm{~kW}$.

In order to investigate the network stability in the frequency domain, the identified $Z_{\text {bus }}$ is plotted in Figure 10 under different load conditions. According to this figure, there is a resonance mode at $118 \mathrm{~Hz}$ which refers to the traction drive input LC filter. This mode is passive and stable in all load conditions. Here, as the load power increases, a bus peaking phenomenon is observed at $32.2 \mathrm{~Hz}$ which is not originated from the passive elements inside the grid. This oscillation mode refers to the dynamic interaction between the AFE 

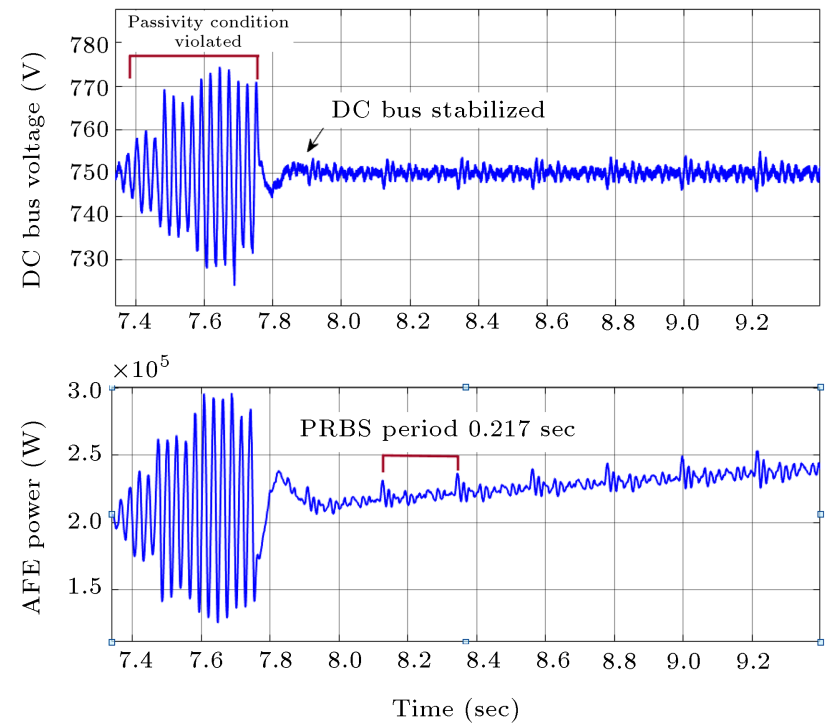

Figure 11. DC bus voltage (top) and power (bottom) at the terminal of the AFE rectifier. Oscillations damped after the injection of the passivizing current.
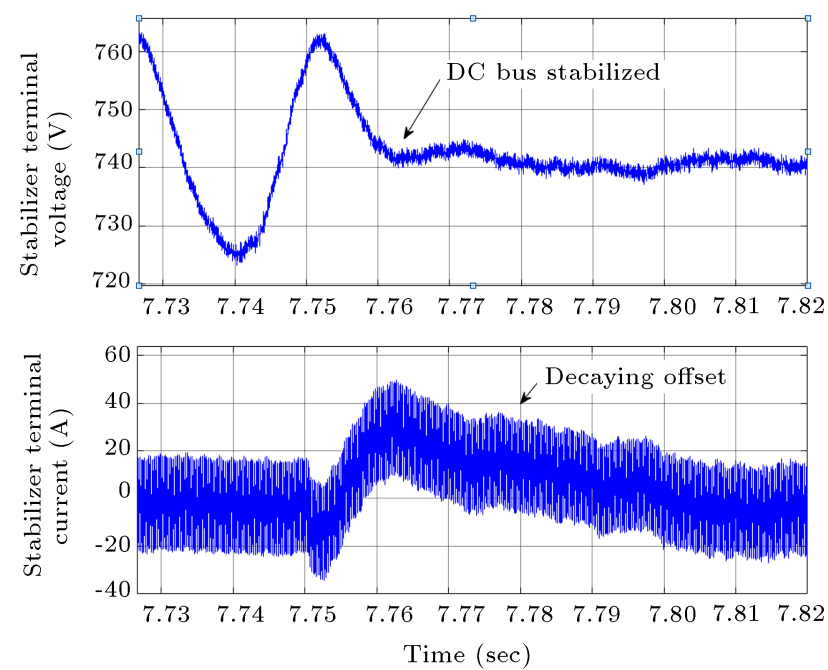

Figure 12. The stabilizer terminal voltage (top) and current (bottom).

rectifier and traction drive. The passivity condition is violated at $200 \mathrm{~kW}$ load power for this oscillation mode.

In the second simulation run, the perturbing and stabilizing circuits of the PSS are both operational. The required $Z_{d a m p}$ is calculated based on the identified $Z_{b u s}$, and the passivizing reference current is applied to the stabilizer current control loop. As shown in Figure 11, the DC bus is stabilized at a load power of $200 \mathrm{~kW}$ after the injection of the passivizing current. In this simulation run, the grid power increases up to $250 \mathrm{~kW}$ to assess the robust stability. In Figure 12, the stabilizer terminal voltage and current are shown at a closer look. At $7.75 \mathrm{~s}$, the passivizing current is injected into the DC bus with an opposite phase to the voltage

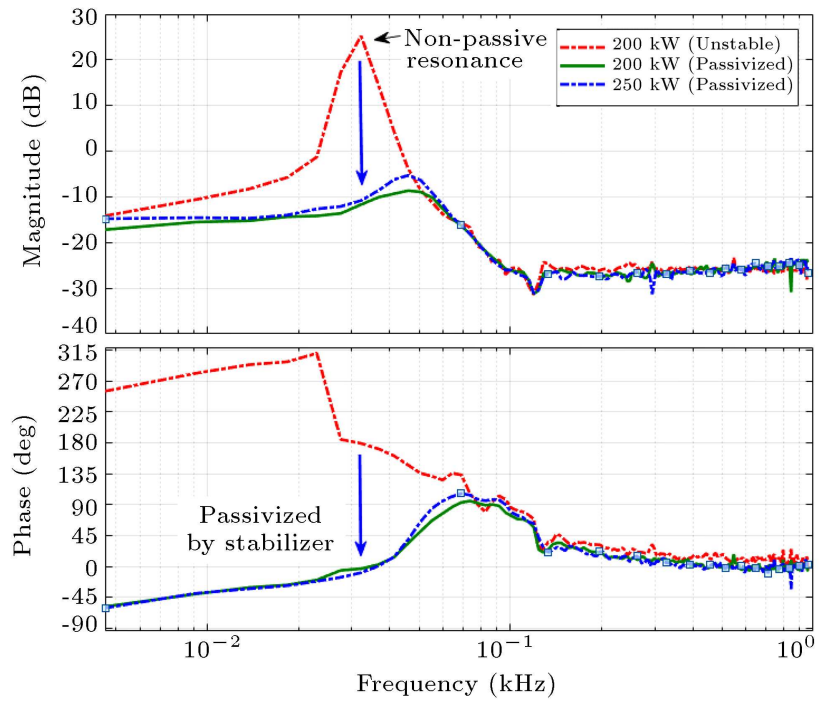

Figure 13. Bode plot of $Z_{\text {bus }}$ before and after the injection of the passivizing current. The power grid is stabilized at the interaction frequency by the PSS.

oscillations. Therefore, the damping power is absorbed from the grid at the interaction frequency; meanwhile, a decaying current offset is also injected into the DC bus, thus balancing the amount of the input and output energy at the PSS port. In order to evaluate the function of the stabilizer from the frequency point of view, the identified $Z_{\text {bus }}$ spectrum before and after the stabilization is plotted in Figure 13 . Prior to stabilization, a resonant peak is observed at $32.2 \mathrm{~Hz}$ with a magnitude of $25 \mathrm{~dB}$ and phase angle of $176^{\circ}$. After the stabilization, the bus impedance magnitude is reduced to $-10 \mathrm{~dB}$ with the passive phase angle $\left(0^{\circ}\right)$ characteristic. The bode plot of the stabilized $Z_{b u s}$ at $250 \mathrm{~kW}$ load power is also plotted in Figure 13 where the sensitivity of the stabilized $Z_{\text {bus }}$ is quite low under the load power variations.

In the next simulation run, the PSS dynamic response is evaluated for the step load changes. The traction motor reference current increased from $100 \mathrm{~A}$ to $300 \mathrm{~A}$ at $2 \mathrm{~s}$ and decreased back to $100 \mathrm{~A}$ at $2.9 \mathrm{~s}$. At first, the PSS is intentionally disabled. Figure 14 presents the DC bus voltage and power in response to the load steps. The DC bus oscillations start right after the load increases from $60 \mathrm{~kW}$ to $300 \mathrm{~kW}$. The simulation is repeated when the PSS is operational. As shown in Figure 15, the DC bus is stabilized at $2.25 \mathrm{~s}$ followed by injecting the passivizing current into the grid. The passivizing current decreases to zero as the voltage oscillations are damped. The DC PSS response is limited to the perturbation and identification process time which is $0.217 \mathrm{~s}$ in this simulation.

\section{Discussion}

The novelty of this paper lies in its application of 

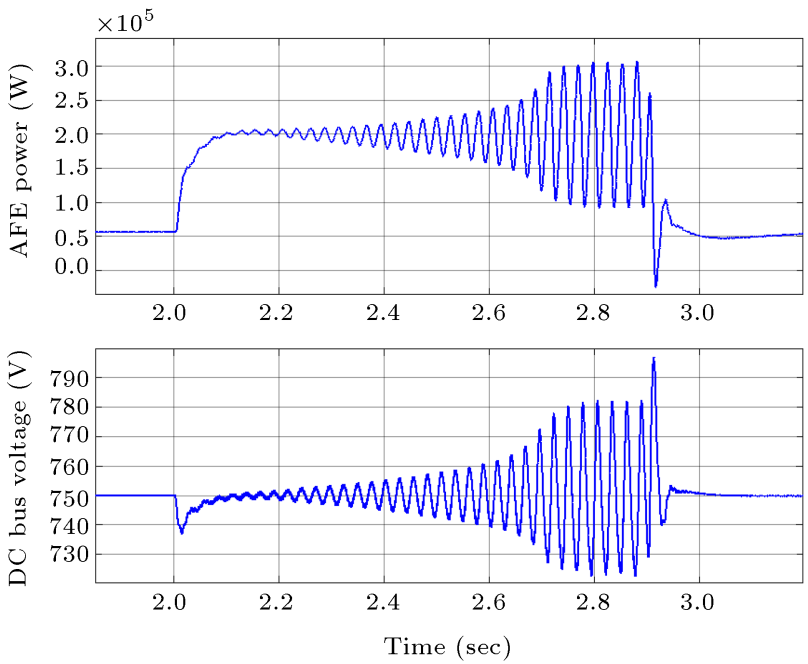

Figure 14. DC bus power (top) and voltage (bottom) responses to the step load variations.

the DC bus stabilization methodology. In contrast to the proposed methods in [16-25], the stabilizing function is implemented by a separate module in a DC grid. Therefore, the control loops and bandwidths of other load or source converters in the grid are not affected by the stabilizing function. Compared to the proposed method in [24], the computational effort is quite low mainly because the control algorithm functions independently of the converter unterminated model (g-parameters). Due to the deadbeat current control, the stabilizer current loop model is fixed and known $\left(z^{-2}\right)$ in the whole frequency ranges. Therefore, the PSS control adaptation is done using only reference current variations.

In $[25,27]$, the bus impedance at resonance frequency is modified by the notch or SOGI filters which, from the circuit point of view, exhibit an RLC branch impedance to the grid. The RLC branch behaves like an inductor in high frequency ranges which can resonate with the network capacitor banks. The proposed stabilizer exhibits an RC branch which is stable in the whole frequency range. In contrast to [25] and [27], the control structure of the proposed stabilizer in the case of the grid resonance frequency variations is fixed. The proposed PSS has a bidirectional power flow capability which injects the absorbed damping power back into the network with a decaying DC current offset. Therefore, there is no need for any battery storage or dissipative dummy loads inside the PSS. Along with the abovementioned advantages, the proposed PSS reduces the network power quality due to the PRBS perturbations.

\section{Conclusion}

The current research combined the concepts of dynamic interaction and passivity together to provide a sufficient condition to detect the instability of a DC power grid, considering the DC bus impedance characteristics. In this regard, a novel Power System Stabilizer (PSS) was proposed which was connected to a grid as a separate module, passivizing the DC bus impedance at interaction frequencies. The stabilizing
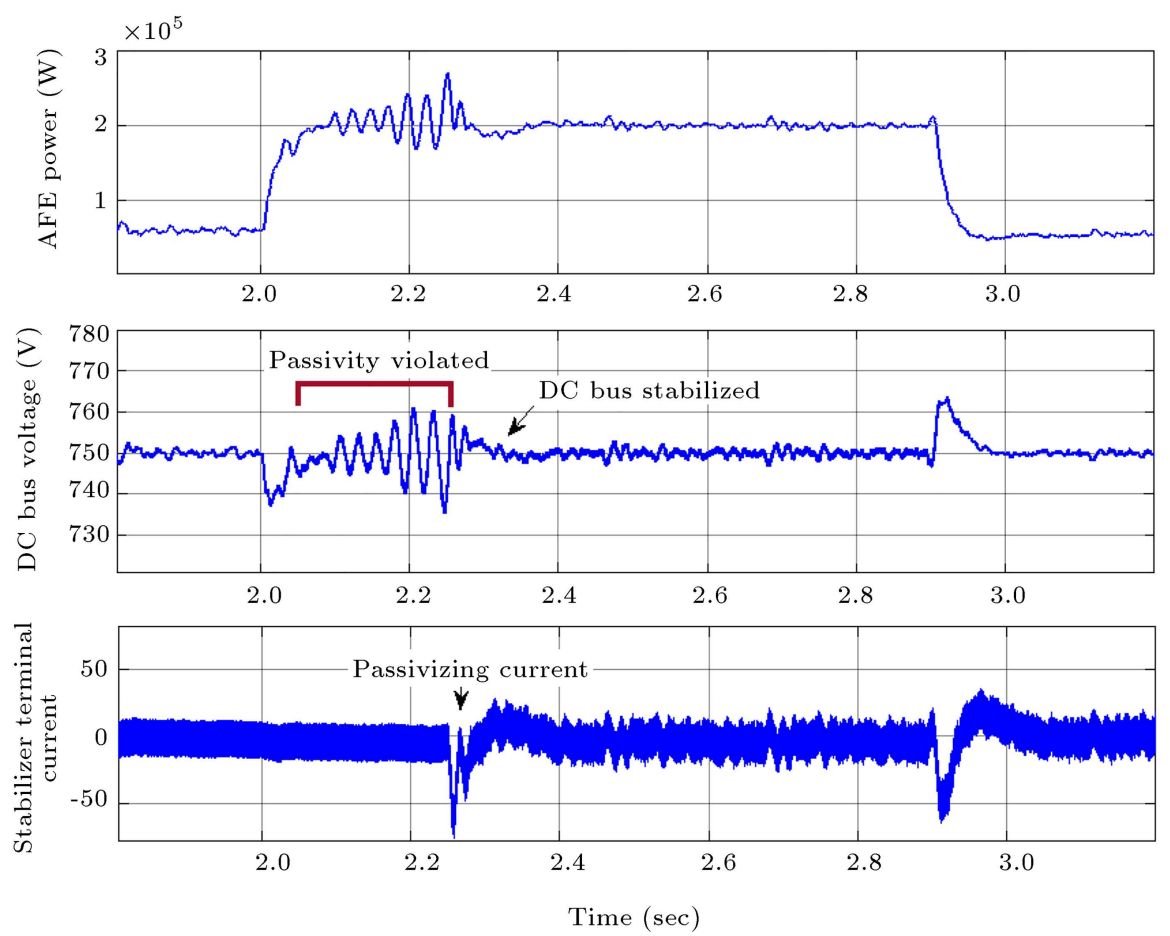

Figure 15. PSS dynamic responses to the step load variations. 
method operated based on local measurements in terms of online identification of the DC bus Impedance. The stabilizer adopts a fixed control structure that emulates a damping impedance with adaptations to the power grid dynamic changes. In addition, several specific definitions were given in this study concerning the stabilizer functionality, circuit topology, modeling, and control designs. The performance and effectiveness of the proposed stabilizer was validated by the simulation results. The obtained results suggested that the proposed stabilizing method was a promising technology for the future DC power grids and microgrids characterized by high penetration of Power Electronic (PE) converters, changing load profiles, and uncertainties.

\section{References}

1. Verdicchio, A., Ladoux, P., Caron, H., et al. "New medium-moltage DC railway electrification system", IEEE Trans. Transp. Electrif., 4(2), pp. 591-604 (2018).

2. Gholdston, E.W., Karimi, K., Lee, F.C., et al. "Stability of large DC power systems using switching converters, with application to the international space station", in IECEC 96. Proceedings of the 31st Intersociety Energy Conversion Engineering Conference, 1, pp. 166-171 (1996).

3. Reed, G.F., Grainger, B.M., Sparacino, A.R., et al. "Ship to grid: medium-voltage dc concepts in theory and practice", IEEE Power Energy Mag., 10(6), pp. 70-79 (2012).

4. De Doncker, R.W. "Power electronic technologies for flexible DC distribution grids", in 2014 International Power Electronics Conference (IPEC-Hiroshima 2014ECCE ASIA), pp. 736-743 (2014).

5. Kazmierkowski, M.P., Krishnan, R., Blaabjerg, F., et al. Control in Power Electronics: Selected Problems, Academic press (2002).

6. Cupeli, M., Ponci, F., Sulligoi, G., et al. "Power flow control and network stability in an all-electric ship", Proc. IEEE, 103(12), pp. 2355-2380 (2015).

7. Mollerstedt, E. and Bernhardsson, B. "Out of control because of harmonics-an analysis of the harmonic response of an inverter locomotive", IEEE Control Syst. Mag., 20(4), pp. 70-81 (2000).

8. Cupelli, M., Zhu, L., and Monti, A. "Why ideal constant power loads are not the worst case condition from a control standpoint", IEEE Trans. Smart Grid, 6(6), pp. 2596-2606 (2014).

9. Middlebrook, R.D. "Input filter considerations in design and application of switching regulators", IAS'r6 (1976).

10. Riccobono, A. and Santi, E. "Comprehensive review of stability criteria for DC power distribution systems", IEEE Trans. Ind. Appl., 50(5), pp. 3525-3535 (2014).
11. Sudhoff, S.D. and Crider, J.M. "Advancements in generalized immittance based stability analysis of DC power electronics based distribution systems", in 2011 IEEE Electric Ship Technologies Symposium, pp. 207212 (2011).

12. Liu, J., Feng, X., Lee, F.C., et al. "Stability margin monitoring for DC distributed power systems via perturbation approaches", IEEE Trans. Power Electron., 18(6), pp. 1254-1261 (2003).

13. Riccobono, A. and Santi, E. "A novel passivity-based stability criterion (PBSC) for switching converter DC distribution systems", in 2012 Twenty-Seventh Annual IEEE Applied Power Electronics Conference and Exposition (APEC), pp. 2560-2567 (2012).

14. Paice, A.D.B. and Meyer, M. "Rail network modelling and stability: the input admittance criterion", in 14th Int. Symp. Math. Theory Netw. Syst., Perpignan, France, pp. 1-6 (2000).

15. Aeberhard, M., Meyer, M., and Courtois, C. "The new standard EN 50388-2, part 2-stability and harmonics", Elektrische Bahnen, 12(1), pp. 28-35 (2014).

16. Mingfei, W.U. and Lu, D.D.C. "Active stabilization methods of electric power systems with constant power loads: a review", J. Mod. Power Syst. Clean Energy, 2(3), pp. 233-243 (2014).

17. Guo, L., Zhang, S., Li, X., et al. "Stability analysis and damping enhancement based on frequency-dependent virtual impedance for DC microgrids", IEEE J. Emerg. Sel. Top. Power Electron., 5(1), pp. 338-350 (2016).

18. Harnefors, L., Bongiorno, M., and Lundberg, S. "Input-admittance calculation and shaping for controlled voltage-source converters", IEEE Trans. Ind. Electron., 54(6), pp. 3323-3334 (2007).

19. Sudhoff, S.D., Corzine, K.A., Glover, S.F., et al. "DC link stabilized field oriented control of electric propulsion systems", IEEE Trans. Energy Convers., 13(1), pp. 27-33 (1998).

20. Sulligoi, G., Bosich, D., Giadrossi, G., et al. "Multiconverter medium voltage DC power systems on ships: Constant-power loads instability solution using linearization via state feedback control", IEEE Trans. Smart Grid, 5(5), pp. 2543-2552 (2014).

21. Cupelli, M., Mirz, M., and Monti, A. "Application of backstepping to MVDC ship power systems with constant power loads", in 2015 International Conference on Electrical Systems for Aircraft, Railway, Ship Propulsion and Road Vehicles (ESARS), pp. 16 (2015).

22. Kondratiev, I. and Dougal, R. "Synergetic control strategies for shipboard DC power distribution systems", in 2007 American Control Conference, pp. 4744-4749 (2007).

23. Magne, P., Nahid-Mobarakeh, B., and Pierfederici, S. "Dynamic consideration of DC microgrids with constant power loads and active damping system-A design method for fault-tolerant stabilizing system", 
IEEE J. Emerg. Sel. Top. Power Electron., 2(3), pp. $562-570$ (2014).

24. Riccobono, A., Siegers, J., and Santi, E. "Stabilizing positive feed-forward control design for a DC power distribution system using a passivity-based stability criterion and system bus impedance identification", in 2014 IEEE Applied Power Electronics Conference and Exposition-APEC 2014, pp. 1139-1146 (2014).

25. Abdollahi, H., Arrua, S., Roinila, T., et al. "A novel dc power distribution system stabilization method based on adaptive resonance-enhanced voltage controller", IEEE Trans. Ind. Electron., 66(7), pp. 5653-5662 (2018).

26. Iyer, V.M., Gulur, S., Bhattacharya, S., et al. "An active voltage stabilizer for a generic dc microgrid", in 2019 IEEE Energy Conversion Congress and Exposition (ECCE), pp. 462-468 (2019).

27. Mohamad, A.M.E.I. and Mohamed, Y.A.R.I. "Investigation and assessment of stabilization solutions for dc microgrid with dynamic loads", IEEE Trans. Smart Grid, 10(5), pp. 5735-5747 (2019).

28. Suntio, T., Dynamic Profile of Switched-Mode Converter: Modeling, Analysis and Control, John Wiley \& Sons (2009).

29. Anderson, B.D.O. and Vongpanitlerd, S. "Network analysis and synthesis: a modern systems theory approach", Courier Corporation, 1, pp. 40-50 (2013).

30. Van Valkenburg, M.E., Introduction to Modern Network Synthesis, John Wiley \& Sons, 1, pp. 62-79 (1966).

31. Godfrey, K. "Perturbation signals for system identification", Prentice Hall International (UK) Ltd. (1993).

32. Martin, D., Santi, E., and Barkley, A. "Wide bandwidth system identification of AC system impedances by applying perturbations to an existing converter", in 2011 IEEE Energy Conversion Congress and Exposition, pp. 2549-2556 (2011).
33. Teodorescu, R., Liserre, M., and Rodriguez, P., Grid Converters for Photovoltaic and Wind Power Systems, 29, John Wiley \& Sons (2011).

34. Miao, B., Zane, R., and Maksimovic, D. "System identification of power converters with digital control through cross-correlation methods", IEEE Trans. Power Electron., 20(5), pp. 1093-1099 (2005).

\section{Biographies}

Ali Asbafkan was born in Isfahan, Iran in 1987. He received his BSc degree in Electrical Engineering from Isfahan University of Technology, Isfahan, Iran in 2010 and MSc degree (with Hons). in Power Electronics and Drives from the University of Isfahan, Isfahan in 2012. He is currently a PhD candidate at the Department of Electrical Engineering, Sharif University of Technology, Tehran, Iran. From 2009 to 2012, he cooperated with the Turbo Design Team in Isfahan University of Technology. His research interests include dynamics and controls of power electronic converters, power quality, electric machines, and drive systems.

Hossein Mokhtari (M'03-SM'14) was born in Tehran, Iran in 1966. He received his BSc degree in Electrical Engineering from Tehran University in 1989, MSc degree in Power Electronics from University of New Brunswick, Fredericton, NB, Canada in 1994, and $\mathrm{PhD}$ degree in Power Electronics/Power Quality from University of Toronto, Toronto, ON, Canada in 1999. From 1989 to 1992, he cooperated with the Consulting Division of Power Systems Dispatching Projects, Electric Power Research Center Institute, Tehran. Since 2000, he has been cooperating with the Department of Electrical Engineering in Sharif University of Technology, Tehran, where he is currently working as a Professor. He is also a senior consultant to several utilities and industries. 